\title{
Consumo de álcool entre estudantes do ensino médio do município de Passos - MG
}

\author{
Alcohol consumption among high school students \\ in the municipality of Passos - MG
}

\author{
Juliana Alvares Duarte Bonini Campos ${ }^{1}$ \\ Jussara de Castro Almeida ${ }^{2}$ \\ Patrícia Petromili Nordi Sasso Garcia ${ }^{1}$ \\ João Bosco Faria ${ }^{2}$
}

${ }^{1}$ Faculdade de Odontologia de Araraquara,

Universidade Estadual de São Paulo (UNESP).

R. Humaitá 1680 14801-903 Araraquara SP. jucampos@foar.unesp.br

${ }^{2}$ Faculdade de Ciências Farmacêuticas,

Universidade Estadual de

São Paulo (UNESP)
Abstract This study identified the pattern of alcohol consumption among high school students in Passos, Minas Gerais State, and its association with socio-demographic factors. Study participants included 1,967 adolescents from public and private educational institutions. The AUDIT questionnaire was used. For data analysis, descriptive statistics, the chi-square test and multiple logistic regression were applied. The average age of participants was $15.84 \pm 1.23$ years. The first contact with alcohol occurred at $13.37 \pm 1.92$ years. Among the adolescents, $30.96 \%$ were teetotalers, $45.76 \%$ had moderate drinking behavior, $16.47 \%$, were risk drinkers, $3.51 \%$ were high-risk drinkers, and $3.31 \%$ possible addicts. There was a significant association between the risk of drinking and sex, the relationship of adolescents with their mothers, work, economic bracket and education of the head of the family. In multivariate analysis, there was a higher risk for adolescent males, as well as workers with a poor relationship with their mothers. It was found that teenagers have early contact with alcohol and high prevalence of risk-related drinking behavior.

Key words Alcoholism, Students, Adolescent health
Resumo Este estudo identificou o padrão de consumo de álcool entre estudantes do ensino médio de Passos (MG) e sua associação com fatores sociodemográficos. Participaram 1.967 adolescentes de instituições públicas e privadas de ensino. Utilizou-se o questionário AUDIT. Realizou-se estatística descritiva, teste de qui-quadrado e regressão logística múltipla. A média de idade dos partici-

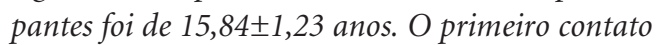
com o álcool ocorreu aos 13,37 $\pm 1,92$ anos. Dos adolescentes, 30,96\% eram abstêmios, 45,76\% apresentaram comportamento de beber moderado, $16,47 \%$ de beber de risco, 3,51\% de alto risco e 3,31\% de possível dependência. Houve associação significativa entre o risco de beber e o sexo, relacionamento do adolescente com a mãe, trabalho, nível econômico e de escolaridade do chefe da família. $\mathrm{Na}$ análise multivariada observou-se maior risco para os adolescentes do sexo masculino, trabalhadores e com ausência de bom relacionamento com a mãe. Verificou-se que os adolescentes apresentaram contato precoce com bebidas alcoólicas e alta prevalência de comportamento de beber de risco. Palavras-chave Alcoolismo, Estudantes, Saúde do adolescente 


\section{Introdução}

O consumo de álcool é apontado como um problema de saúde pública em função de sua magnitude, transcendência e existência de métodos preventivos e de controle ${ }^{1,2}$, sendo que a transição do comportamento de beber moderado ao problemático ocorre de forma lenta ${ }^{3}$. Além disso, o consumo de bebidas alcoólicas tem ocorrido em idades precoces antecipando e agravando os riscos à saúde e a dependência desta substân$\mathrm{cia}^{3}$. Portanto, estudos que avaliem esse consumo e seus fatores associados são importantes.

Segundo Vizzolto ${ }^{4}$, Andersen et al. ${ }^{5}$ e o Departamento de Adolescência da Sociedade Brasileira de Pediatria ${ }^{6}$, a adolescência é uma etapa onde o indivíduo está mais susceptível ao desenvolvimento de comportamento de beber de risco devido às grandes mudanças fisiológicas e psicológicas desta fase associadas ao contexto social em que se vive.

Em Cartagena, Espanha, López et al. ${ }^{7}$, detectaram uma prevalência de consumo de álcool entre adolescentes de $83,40 \%$, sendo a média de idade do primeiro contato com a bebida de $15,84 \pm 1,21$ anos. Gallego et al. ${ }^{8}$, verificaram que $93,44 \%$ dos estudantes do ensino médio de Toledo, Espanha, já experimentaram bebidas alcoólicas, 50,00\% ingeriram álcool antes dos 14 anos e 52,00\% apresentaram episódios de embriaguez.

Caetano e Babor ${ }^{9}$, verificaram nos Estados Unidos que $4,60 \%$ dos adolescentes de 12 a 17 anos de idade eram dependentes de álcool.

No Brasil, o V Levantamento sobre o consumo de drogas psicotrópicas entre estudantes do ensino fundamental e médio da rede pública de ensino nas 27 capitais brasileiras, detectou uma prevalência de consumo e de dependência de bebidas alcoólicas de $65,20 \%$ e $6,70 \%$, respectivamente. De acordo com os adolescentes, em média, o primeiro contato com o álcool, foi aos $12,50 \pm 2,10$ anos de idade. Em Belo Horizonte (MG), o uso na vida e o uso frequente de álcool foi relatado por $67,80 \%$ e $14,20 \%$ dos participantes, respectivamente ${ }^{10}$.

Vieira et al. ${ }^{11}$ revelaram uma prevalência de $62,20 \%$ de consumo de álcool entre os alunos de escolas públicas e particulares do município de Paulínia (SP). Participaram do estudo 1.990 alunos, com idades entre 11 e 21 anos. Dos participantes, $17,30 \%$ relataram episódios de abuso de bebidas alcoólicas. Os autores destacam que quando os adolescentes bebem, tendem a fazê-lo de forma exagerada, aumentando assim, o risco de problemas sociais e de saúde.
Frente à relevância do alcoolismo, no cenário da saúde pública brasileira e considerando a importância do diagnóstico precoce para a implantação de políticas públicas voltadas para a prevenção e tratamento desta condição, o presente estudo teve por objetivo identificar o padrão de consumo de álcool entre estudantes do ensino médio do município de Passos (MG) e sua associação com o sexo, idade, relacionamento familiar e aspectos pessoais como religião, prática esportiva e trabalho.

\section{Material e métodos}

Foi realizado um estudo observacional do tipo transversal com delineamento amostral probabilístico estratificado segundo o tipo de instituição de ensino (pública e privada), número de estudantes por escola, série cursada e sexo.

Os dados referentes ao total de adolescentes matriculados no ensino médio no município de Passos (MG) foram obtidos junto à Secretaria da Educação da cidade, de modo que, este valor foi de 4.805 escolares, sendo 4.153 matriculados em escolas públicas e $652 \mathrm{em}$ instituições privadas.

No pré-teste do delineamento amostral duas escolas particulares se recusaram a participar da pesquisa e, portanto, foram excluídas do cálculo de tamanho amostral. Assim, considerou-se o número de 505 escolares provenientes da escola privada.

O nível de significância adotado foi de 5,00\% e considerou-se como estimativa preliminar da verdadeira proporção de dependentes de álcool o valor de 7,90\%, encontrado por Galduróz et al. ${ }^{10}$ para Belo Horizonte (MG). A margem relativa de erro foi fixada em $20,00 \%$.

Nestas condições, o tamanho amostral mínimo ficou estimado em 883 indivíduos matriculados em escolas públicas e 349 nas instituições privadas. Tendo-se admitido um absenteísmo da ordem de $20,00 \%$, o tamanho da amostra foi corrigido e passou a ser de 1.104 e 437 para escolas públicas e privadas, respectivamente.

Cabe ressaltar que participaram do estudo apenas os adolescentes cujos responsáveis preencheram e concordaram com o Termo de Consentimento Livre e Esclarecido.

Para identificação de desordens devido ao álcool utilizou-se o questionário AUDIT desenvolvido pela Organização Mundial de Saúde (OMS) composto por 10 questões objetivas que permitem respostas com pesos pré-estabelecidos de 0 a $4^{12}$. O somatório dos pesos de cada questão 
indica a classificação de cada indivíduo frente ao consumo de bebidas alcoólicas.

Neste estudo, utilizou-se a versão em português do AUDIT validada por Méndez ${ }^{13}$ e posteriormente por Lima et al. ${ }^{14}$. Para utilização desta versão em adolescentes, Almeida e Campos ${ }^{15}$ realizaram estudos de confiabilidade e verificaram excelente consistência interna e reprodutibilidade $(\alpha=0,7667, \kappa=0,92)$ do AUDIT.

Para caracterização da amostra foram levantadas ainda informações referentes à idade, sexo, relacionamento familiar, presença ou não de religião, prática esportiva, trabalho, nível econômico, nível de escolaridade do chefe da família e idade de primeiro contato com o álcool.

A idade foi analisada em anos completos, a prática de esportes, o trabalho, a religião e o consumo de bebidas alcoólicas pelos pais foi avaliada em escala dicotômica e para relacionamento familiar adotou-se uma escala Likert de 4 pontos.

Para avaliação do nível socioeconômico e de escolaridade utilizou-se a escala proposta pela Associação Brasileira do Instituto de Pesquisa de Mercado ${ }^{16}$.

Realizou-se estatística descritiva das variáveis.

Para o estudo de associação entre o comportamento de consumo de bebidas alcoólicas e as variáveis de interesse utilizou-se o teste de quiquadrado $\left(\chi^{2}\right)$. Para tanto, os indivíduos foram agrupados, segundo a pontuação total do AUDIT, em "risco ausente" ( $<8$ pontos) e "risco presente" ( $\geq 8$ pontos). A variável nível de escolaridade do chefe da família foi categorizada em "baixo" quando a escolaridade atingiu apenas até colegial incompleto e "alto" a partir de colegial completo. As variáveis de relacionamento com o pai, com a mãe e entre os pais para estudo de associação, foram dicotomizadas em "bom relacionamento" e "demais categorias". Adotou-se nível de significância de 5,00\%. Os dados que apresentaram significância estatística ou $\mathrm{p} \leq 0,15$ compuseram a análise de regressão logística múltipla.

Este trabalho foi realizado após a aprovação do Comitê de Ética em Pesquisa da Faculdade de Ciências Farmacêuticas de Araraquara.

\section{Resultados}

Participaram do estudo 1.967 indivíduos, estudantes do ensino médio, sendo, 1.611 (81,90\%) matriculados em escolas públicas e $356(18,10 \%)$ em instituições privadas, atingindo, portanto, o tamanho amostral mínimo calculado. De acordo com a série do ensino médio, $41,38 \%$ dos participantes frequentavam o $1^{\circ}$ ano, $31,83 \%$ o $2^{\circ}$ ano e $26,79 \%$ o $3^{\circ}$ ano, preservando, portanto, a representatividade no município de Passos (MG).

A média de idade dos estudantes foi de 15,84 1 1,23 anos, com mínimo de 13 e máximo de 29 anos, sendo 57,38\% do sexo feminino. De acordo com os relatos dos estudantes, o primeiro contato com o álcool ocorreu em média aos $13,37 \pm 1,92$ anos. O número de pessoas que residem no domicílio com o adolescente foi em média de 4,36 1,27, com o número mínimo de 1 e o máximo de 13 moradores por casa, observou-se que, 61,69\% das residências apresentavam quatro ou menos moradores.

A classificação dos estudantes frente ao consumo de bebidas alcoólicas segundo a pontuação total do AUDIT encontra-se na Tabela 1.

Observa-se que a maioria dos participantes consome bebida alcoólica e 23,28\% apresentaram comportamento de beber de risco.

A distribuição dos estudantes segundo o consumo de risco de bebidas alcoólicas (ausente, presente) e as variáveis sociodemográficas de interesse estão expostos na Tabela 2 .

A maioria dos participantes relatou praticar algum esporte (66,62\%), não trabalhar $(65,16 \%)$, seguir alguma religião $(92,06 \%)$, ter um bom relacionamento com o pai $(79,77 \%)$, com a mãe $(93,74 \%)$ e que os pais apresentavam um bom relacionamento entre si $(77,08 \%)$. Chama atenção o relato de que a maioria dos pais ingere bebidas alcoólicas $(65,19 \%)$. Verificou-se também que 54,55\% possuem baixo nível econômico e $57,14 \%$ dos chefes da família apresentam baixa escolaridade.

Observou-se associação significativa entre o risco de beber e o sexo $\left(\chi^{2}=9,640, \mathrm{p}=0,002\right)$, relacionamento do adolescente com a mãe $\left(\chi^{2}=14,603, \mathrm{p}=0,001\right)$, trabalho $\left(\chi^{2}=8,754\right.$, $\mathrm{p}=0,003)$, nível econômico $\left(\chi^{2}=4,074, \mathrm{p}=0,044\right)$ e escolaridade do chefe da família $\left(\chi^{2}=8,754\right.$, $\mathrm{p}=0,003)$.

Tabela 1. Padrão de consumo de bebida alcoólica segundo proposta de Babor et $\mathrm{al}^{12}$. Passos - MG, 2008.

\begin{tabular}{lrrc}
\hline \multicolumn{1}{c}{ Classificação } & n & \multicolumn{1}{c}{$\%$} & \multicolumn{1}{c}{ IC $_{95 \%}$} \\
\hline Abstêmios & 609 & 30,96 & $28,92-33,06$ \\
Beber moderado & 900 & 45,76 & $43,54-47,99$ \\
Padrão de beber de risco & 324 & 16,47 & $14,86-18,19$ \\
Padrão de beber de alto risco & 69 & 3,51 & $2,74-4,42$ \\
Possível dependência de álcool & 65 & 3,30 & $2,56-4,19$ \\
Total & 1.967 & 100,00 & \\
\hline
\end{tabular}


Tabela 2. Distribuição dos estudantes segundo o consumo de beber de risco (ausente, presente) e as variáveis sóciodemográficas de interesse. Passos - MG, 2008.

\begin{tabular}{|c|c|c|c|c|c|}
\hline \multirow[b]{2}{*}{ Características sóciodemográficas } & \multicolumn{2}{|c|}{ Risco } & \multirow[b]{2}{*}{ Total } & \multirow[b]{2}{*}{$\chi^{2}$} & \multirow[b]{2}{*}{$\mathbf{p}$} \\
\hline & Ausente & Presente & & & \\
\hline \multicolumn{6}{|l|}{ Sexo } \\
\hline Masculino & 614 & 224 & 838 & & \\
\hline Feminino & 894 & 234 & 1.128 & 9,640 & $0,002^{*}$ \\
\hline Total & & & 1.966 & & \\
\hline \multicolumn{6}{|l|}{ Série do ensino médio } \\
\hline $1^{\circ}$ ano & 637 & 177 & 814 & & \\
\hline $2^{\circ}$ ano & 469 & 157 & 626 & & \\
\hline $3^{\circ}$ ano & 403 & 124 & 527 & 2,228 & 0,328 \\
\hline Total & & & 1.967 & & \\
\hline \multicolumn{6}{|l|}{ Tipo de escola } \\
\hline Pública & 1.243 & 368 & 1.611 & & \\
\hline Particular & 266 & 90 & 356 & 0,970 & 0,325 \\
\hline Total & & & 1.967 & & \\
\hline \multicolumn{6}{|l|}{ Quantas pessoas moram na sua casa? } \\
\hline Menor ou igual a 4 pessoas & 924 & 287 & 1.211 & & \\
\hline Mais de 4 pessoas & 581 & 171 & 752 & 0,239 & 0,625 \\
\hline Total & & & 1.963 & & \\
\hline \multicolumn{6}{|l|}{ Você pratica esporte? } \\
\hline Não & 502 & 154 & 656 & & \\
\hline Sim & 1.006 & 303 & 1.309 & 0,026 & 0,871 \\
\hline Total & & & 1.965 & & \\
\hline \multicolumn{6}{|l|}{ Você trabalha? } \\
\hline Não & 1.009 & 272 & 1.281 & & \\
\hline Sim & 499 & 186 & 685 & 8,754 & $0,003^{*}$ \\
\hline Total & & & 1.966 & & \\
\hline \multicolumn{6}{|l|}{ Você segue alguma religião? } \\
\hline Não & 110 & 46 & 156 & & \\
\hline Sim & 1.398 & 411 & 1.809 & 3,685 & 0,055 \\
\hline Total & & & 1.965 & & \\
\hline \multicolumn{6}{|l|}{ Como é o relacionamento com o seu pai? } \\
\hline Não tenho pai & 91 & 23 & 114 & & \\
\hline Bom & 1.210 & 359 & 1.569 & & \\
\hline Regular & 116 & 45 & 161 & & \\
\hline Ruim & 32 & 17 & 49 & & \\
\hline Não tenho contato & 60 & 14 & 74 & 0,706 & 0,401 \\
\hline Total & & & 1.967 & & \\
\hline \multicolumn{6}{|l|}{ Como é o relacionamento com a sua mãe? } \\
\hline Não tenho mãe & 18 & 8 & 26 & & \\
\hline Bom & 1.431 & 412 & 1.843 & & \\
\hline Regular & 51 & 30 & 81 & & \\
\hline Ruim & 4 & 4 & 8 & & \\
\hline Não tenho contato & 4 & 4 & 8 & 14,603 & $0,001^{*}$ \\
\hline Total & & & 1.966 & & \\
\hline Como é o relacionamento entre seus pais & & & & & \\
\hline Não tenho pai & 89 & 21 & 110 & & \\
\hline Bom & 1.167 & 336 & 1.503 & & \\
\hline Regular & 140 & 52 & 192 & & \\
\hline Ruim & 55 & 26 & 81 & & \\
\hline Não tenho contato & 45 & 19 & 64 & 3,153 & 0,076 \\
\hline Total & & & 1.950 & & \\
\hline Seus pais tomam bebidas alcoólicas? & & & & & \\
\hline Não & 535 & 149 & 684 & & \\
\hline Sim & 972 & 309 & 1.281 & 1,364 & 0,243 \\
\hline Total & & & 1.965 & & \\
\hline Nível econômico $\left(\mathrm{SM}^{* *}\right)$ & & & & & \\
\hline $\mathrm{A}$ e B $\quad 10 \vdash+$ & 667 & 227 & 894 & & \\
\hline $\mathrm{C}, \mathrm{D}$ e $\mathrm{E} \quad 0 \vdash 10$ & 842 & 231 & 1.073 & 4,074 & $0,044^{*}$ \\
\hline Total & & & 1.967 & & \\
\hline Escolaridade do chefe da família & & & & & \\
\hline Baixa & 883 & 241 & 1.124 & & \\
\hline Alta & 626 & 217 & 843 & 8,754 & $0,003^{*}$ \\
\hline Total & & & 1.967 & & \\
\hline
\end{tabular}

*Diferença estatística significante para $\alpha=0,05 .{ }^{* *}$ SM: Salários mínimos 
O modelo multivariado encontra-se na $\mathrm{Ta}-$ bela 3 .

Observa-se que o risco de consumir bebidas alcoólicas é significativamente maior nos estudantes do sexo masculino $\left(\mathrm{OR}=1,292 ; \mathrm{IC}_{95 \%}\right.$ =1,038-1,608), com ausência de bom relacionamento com a mãe $\left(\mathrm{OR}=2,009 ; \mathrm{IC}_{95 \%}=1,322\right.$ $3,052)$ e que eram trabalhadores ( $\mathrm{OR}=1,359$; $\left.\mathrm{IC}_{95 \%}=1,085-1,703\right)$.

Na Tabela 4 estão apresentadas as frequências das respostas ao Teste de Identificação de Desordens Devido ao Álcool (AUDIT).

Dos estudantes, $31,50 \%$ relataram nunca ter consumido bebida alcoólica no último ano. Nas ocasiões em que consumiram bebida alcoólica no último ano, 48,33\% relataram o consumo de 1 ou 2 doses, enquanto $28,33 \%$ apontaram consumir acima de 5 doses. Quanto à frequência de consumo de seis ou mais doses em uma mesma ocasião, o que aponta para o comportamento de beber em "binge", observa-se que $11,37 \%$ relataram realizar este comportamento uma vez por semana e $1,65 \%$ o fazia todos ou quase todos os dias. Dos participantes, $7,51 \%$ relataram que não foram capazes de controlar a quantidade de bebida a ser ingerida depois de começar, com uma frequência de uma vez por semana ou mais e $3,12 \%$ não conseguiram cumprir compromissos por causa do consumo de bebidas.

Outro dado que merece destaque, é que 10,34\% dos estudantes afirmaram já ter precisado beber pela manhã para se sentir melhor. O sentimento de culpa ou remorso depois de beber foi observado em $19,98 \%$ dos entrevistados, $25,60 \%$ dos adolescentes relataram não se lembrar do que aconteceu na noite anterior por causa da bebida e $4,83 \%$ relataram ter se prejudicado ou prejudicado outra pessoa, devido à bebida. A preocupação de parentes, amigos, médico ou profissional da saúde por causa do consumo de bebida alcoólica foi apontado por $9,86 \%$ dos estudantes.

\section{Discussão}

Cabe ressaltar que, a comparação dos dados sobre o padrão de consumo de álcool entre adolescentes desta pesquisa torna-se difícil devido à escassez de estudos epidemiológicos, com adequada validade externa, que utilizam o AUDIT. Entretanto, é importante destacar que este instrumento é considerado confiável e válido, sendo recomendado pela Organização Mundial de Saúde para o rastreamento do consumo de bebidas alcoólicas inclusive entre adolescentes ${ }^{12}$. Outro aspecto que merece destaque é a adequada consistência interna $(\alpha=0,77)$ e reprodutibilidade $(\kappa=0,92)$ deste instrumento, já observadas em estudo anterior realizado por Almeida e Campos ${ }^{15}$.

No presente estudo verificou-se que a média de idade de primeiro contato com o álcool ocorreu aos $13,37 \pm 1,92$ anos semelhante ao encontrado em estudos nacionais e internacionais ${ }^{7,10,17-21}$. No entanto, apesar de o valor obtido neste estudo concordar com a literatura, torna-se importante salientar que esta idade observada está abaixo da mínima legal para a aquisição e consumo desta substância, o que é preocupante pois de acordo com Andersen et al. ${ }^{5}$, Caetano e Babor ${ }^{9}$, Vieira et al. ${ }^{11}$, McCarty et al..$^{22}$, e Vorst et al. ${ }^{23}$, quanto mais cedo ocorrer a exposição ao álcool, maiores são as chances dos indivíduos de consumi-lo excessivamente ao longo da vida.

Frente ao padrão de consumo de bebidas alcoólicas segundo a pontuação total do AUDIT (Tabela 1), observou-se uma elevada prevalência de consumo de álcool pelos adolescentes (59,04\%), inferior, porém, à encontrada por Galduróz et al. ${ }^{10}$

Tabela 3. Modelo da regressão logística múltipla para o risco de beber e as variáveis sócio-demográficas de interesse. Passos - MG, 2008.

\begin{tabular}{lcccc}
\hline \multicolumn{1}{c}{ Variáveis } & Coeficiente & OR & IC $_{95 \%}$ & p \\
\hline Intercepto & $-1,472$ & - & - & - \\
Sexo & 0,256 & 1,292 & $1,038-1,608$ & $0,022^{*}$ \\
Trabalho & 0,307 & 1,359 & $1,085-1,703$ & $0,008^{*}$ \\
Religião & $-0,207$ & 0,813 & $0,559-1,182$ & 0,278 \\
Relacionamento com a mãe & 0,698 & 2,009 & $1,322-3,052$ & $0,001^{*}$ \\
Relacionamento entre os pais & 0,111 & 1,117 & $0,863-1,446$ & 0,400 \\
Nível econômico & 0,173 & 1,189 & $0,915-1,544$ & 0,194 \\
Escolaridade & 0,191 & 1,211 & $0,933-1,571$ & 0,151 \\
\hline
\end{tabular}

\footnotetext{
Diferença estatística significante para $\alpha=0,05$.
} 
Tabela 4. Distribuição dos participantes segundo as respostas ao Teste de Identificação de Desordens Devido ao Álcool (AUDIT). Passos - MG, 2008.

\begin{tabular}{|c|c|c|c|c|c|c|}
\hline \multirow[b]{2}{*}{ Questões } & \multicolumn{6}{|c|}{ Respostas } \\
\hline & A & B & $\mathrm{C}$ & D & E & Total \\
\hline $\begin{array}{l}{ }^{*} 1 \text { - Quantas vezes você toma } \\
\text { bebida de álcool? }\end{array}$ & $619(31,50)$ & $734(37,35)$ & $366(18,63)$ & $144(7,33)$ & $102(5,19)$ & 1.965 \\
\hline $\begin{array}{l}{ }^{*} 2 \text { - Nas ocasiões em que bebe, } \\
\text { quantas doses, copos ou garrafas } \\
\text { você costuma tomar? }\end{array}$ & $650(48,33)$ & $314(23,35)$ & $165(12,27)$ & $83(6,17)$ & $133(9,89)$ & 1.345 \\
\hline $\begin{array}{l}{ }^{* *} 3 \text { - Quantas vezes você toma } \\
\text { "seis ou mais doses" em uma } \\
\text { ocasião? }\end{array}$ & $604(45,18)$ & $387(28,95)$ & $172(12,86)$ & $152(11,37)$ & $22(1,65)$ & 1.337 \\
\hline $\begin{array}{l}{ }^{* *} 4 \text { - Com que frequência, } \\
\text { durante o último ano, você } \\
\text { achou que não seria capaz de } \\
\text { controlar a quantidade de bebida } \\
\text { depois de começar? }\end{array}$ & $993(73,88)$ & $168(12,50)$ & $82(6,10)$ & $57(4,24)$ & $44(3,27)$ & 1.344 \\
\hline $\begin{array}{l}\text { durante o último ano, você não } \\
\text { conseguiu cumprir com algum } \\
\text { compromisso por causa da } \\
\text { bebida? }\end{array}$ & $1.165(86,55)$ & $100(7,43)$ & $39(2,90)$ & $17(1,26)$ & $25(1,86)$ & 1.346 \\
\hline $\begin{array}{l}\text { *** } 6 \text { - Com que frequência, } \\
\text { durante o último ano, depois } \\
\text { de ter bebido muito, você } \\
\text { precisou beber pela manhã } \\
\text { para se sentir melhor? }\end{array}$ & $1.206(89,67)$ & $76(5,65)$ & $25(1,86)$ & $18(1,34)$ & $20(1,49)$ & 1.345 \\
\hline $\begin{array}{l}\text { *** } 7 \text { - Com que frequência, } \\
\text { durante o último ano, você } \\
\text { sentiu culpa ou remorso } \\
\text { depois de beber? }\end{array}$ & $1.077(80,01)$ & $178(13,22)$ & $54(4,01)$ & $12(0,89)$ & $25(1,86)$ & 1.346 \\
\hline $\begin{array}{l}\text { durante o último ano, você não } \\
\text { conseguiu se lembrar do que } \\
\text { aconteceu na noite anterior por } \\
\text { causa da bebida? }\end{array}$ & $1.003(74,41)$ & $213(15,80)$ & $57(4,23)$ & $28(2,08)$ & $47(3,49)$ & 1.348 \\
\hline $\begin{array}{l}{ }^{* * *} 9 \text { - Alguma vez na vida você } \\
\text { ou alguma outra pessoa já se } \\
\text { machucou, se prejudicou por } \\
\text { causa de você ter bebido? }\end{array}$ & $1.751(89,06)$ & & $120(6,10)$ & & $95(4,83)$ & 1.966 \\
\hline $\begin{array}{l}{ }^{* * *} 10 \text { - Alguma vez na vida } \\
\text { algum parente, amigo, médico } \\
\text { ou outro profissional da saúde já } \\
\text { se preocupou com você por causa } \\
\text { de bebida ou lhe disse para parar } \\
\text { de beber? }\end{array}$ & $1.646(83,68)$ & & $127(6,46)$ & & $194(9,86)$ & 1.967 \\
\hline
\end{tabular}

"A: nunca, B: uma vez por mês ou menos, C: duas a quatro vezes por mês, D: duas a três vezes por semana, E: quatro ou mais vezes por semana. ${ }^{* *}$ A: 1 ou 2 “doses”, B: 3 ou 4 “doses", C: 5 ou 6 “doses”, D: 7 a 9 “doses”, E: 10 ou mais “doses”. "** A: nunca, B: uma vez por mês ou menos, C: uma vez ao mês, D: uma vez por semana, E: todos os dias ou quase todos. ${ }^{* * *}$ A: não, C: sim, mas não no último ano, E: sim, durante o último ano.

no levantamento nacional $(65,20 \%)$ e em especial para o município de Belo Horizonte (MG) $(67,20 \%)$. Resultados superiores também foram encontrados por López et al. ${ }^{7}$ em Catargena (83,40\%), Lama et al. ${ }^{24}$ em Córdoba $(88,90 \%)$,
Andersen et al..$^{5}$ na Dinamarca (80,00\%), Molina et al. ${ }^{17}$ em Córdoba $(80,20 \%)$, Mateos et al. ${ }^{19} \mathrm{em}$ Lanzarote $(69,20 \%)$, Gallego et al. ${ }^{8}$ em Toledo (93,44\%), Souza et al..$^{20}$ em Cuiabá $(71,30 \%)$ e Vieira et al. ${ }^{11}$ em Paulínia (62,20\%). 
O consumo problemático de bebidas alcoólicas foi encontrado em $6,81 \%$ dos estudantes. Tal fato sugere uma precocidade deste comportamento e merece atenção das autoridades governamentais e da sociedade. O consumo excessivo e a dependência de bebidas alcoólicas é um problema de saúde pública e afeta não somente o indivíduo, mas também a família e a comunidade ${ }^{25}$, trazendo sérias consequências como o desenvolvimento de tolerância, acidentes automobilísticos, envolvimento em brigas, agressões, quedas, maior exposição a doenças sexualmente transmissíveis, gravidez, prejuízos acadêmicos, uso de outras substâncias psicoativas, desordens mentais, patologias no fígado, problemas no aparelho digestivo, no sistema cardiovascular, polineurite alcoólica e até mesmo a morte $e^{1,12,21,26,27}$.

Assim como a idade de início para o consumo de álcool é considerada um importante fator preditor para o desenvolvimento de um comportamento de consumo abusivo e/ou dependência desta substância na idade adulta, outros fatores como as características sociodemográficas também podem ser considerados para o seu desencadeamento.

$\mathrm{Na}$ Tabela 2, pode-se notar a associação significativa entre o consumo de risco de bebidas alcoólicas e o sexo, a presença de trabalho, o relacionamento com a mãe, o nível econômico e a escolaridade do chefe da família.

Observou-se um maior risco de consumo de bebidas alcoólicas entre os estudantes do sexo masculino de Passos (MG) (Tabela 3) corroborando com os achados do levantamento nacional realizado por Galduróz et al. ${ }^{10}$ e outros estudos como os de Andersen et al. ${ }^{5}$, Molina et al. ${ }^{17} \mathrm{e}$ Colby et al. ${ }^{28}$ Entretanto, segundo Laranjeira e Pinsky ${ }^{29}$ a diferença entre os sexos vem diminuindo com o passar dos anos, devido às mudanças sociais decorrentes das conquistas femininas.

A associação significativa entre o risco de consumo de bebidas alcoólicas e o trabalho confirma resultados já observados por Souza et al..$^{20} \mathrm{e}$ Galduróz et al. ${ }^{10}$ que apontam maior prevalência de alcoolismo e uso "pesado" de álcool entre os adolescentes trabalhadores, o que pode estar relacionado à independência financeira para a aquisição de álcool, convivência com adultos que utilizam esta substância em reuniões de lazer ou pelo estresse causado pelo trabalho.

Com relação ao relacionamento do adolescente com a mãe, detectou-se que aqueles indivíduos que relataram ter um bom relacionamento apresentaram um menor risco para o consumo de álcool (Tabela 3), sendo o mesmo observado por
Galduróz et al. ${ }^{10}$ De acordo com Oliveira et al. ${ }^{25} \mathrm{e}$ Schinkerl e Minayo ${ }^{30}$, este fato pode ser explicado por ser a figura materna altamente representativa dentro do núcleo familiar, sendo, de forma geral, a principal responsável pela transmissão de valores vigentes na sociedade. Schenker ${ }^{1}$ Minayo ${ }^{30}$ destacam também a importância das trocas afetivas relacionadas com a mãe que podem ser decisórias no modo de agir dos indivíduos.

Apesar de Gallego et al. ${ }^{8}$ e Vorst et al. ${ }^{23}$, apontarem que um bom relacionamento familiar e comportamento dos pais frente ao consumo de bebidas alcoólicas possam atuar como um fator protetor para o consumo desta substância, nas Tabelas 2 e 3 nota-se que apenas o relacionamento com a mãe apresentou efeito significativo no comportamento de risco, neste estudo.

O consumo de bebidas alcoólicas segundo o nível econômico e a escolaridade do chefe da família têm sido estudado, entretanto, a literatura é controversa. Colby et al. ${ }^{28}$ e Ruiz e Andrade ${ }^{31}$, apontam a situação socioeconômica baixa como sendo um fator de risco para o consumo de bebidas alcoólicas. Já nos estudos de Souza et al. ${ }^{20} \mathrm{e}$ Silva et al. ${ }^{32}$, os escolares com renda familiar mais alta foram os que apresentaram maior risco de consumo de drogas e álcool. A baixa escolaridade da família é apontada por Ruiz e Andrade ${ }^{31}$ como uma dificuldade para orientar os filhos em relação ao consumo de substâncias psicotrópicas, e consequentemente, deve atuar como fator de risco para o consumo de bebidas alcoólicas. Nos adolescentes de Passos (MG), verificou-se (Tabela 3 ) que estas variáveis não estiveram significativamente associadas ao risco de consumo de bebidas alcoólicas.

Outro aspecto a ser ressaltado também é que apesar da religiosidade ser apontada por Silva et al. ${ }^{32}$, Dalgalarrondo et al. ${ }^{33}$ e Kozaryn-Okulicz e Borucka $^{34}$ como sendo um fator protetor para o consumo de álcool e de outras drogas, nesta pesquisa (Tabela 3) isto não foi observado, o que pode ter ocorrido pelo fato do estudante ter afirmado possuir uma religião mas não necessariamente ser praticante da mesma.

Na Tabela 4 chama a atenção o consumo de grande quantidade de bebida alcoólica em uma única ocasião relatada por $13,02 \%$ dos adolescentes (questão 3), o que caracteriza, o comportamento de beber em "binge". Andersen et al. ${ }^{5}$, Vieira et al. ${ }^{11}$ e MacCarty et al. ${ }^{22}$ alertam que este tipo de comportamento traz graves consequências ao indivíduo, como intoxicação e predisposição a continuidade de uso e alcoolismo na idade adulta. 
Torna-se difícil a comparação dos achados referentes às respostas do AUDIT (Tabela 4) com a literatura, uma vez que, como já dito anteriormente, são poucos ainda os trabalhos que utilizam este instrumento para detectar o consumo de álcool entre estudantes do ensino médio.

Frente à realidade aqui verificada fica evidenciada a importância da detecção precoce do consumo de bebidas alcoólicas pelos adolescentes, bem como sua associação com os fatores sociodemográficos, para o correto direcionamento de estratégias de prevenção e combate ao consumo de risco e ao alcoolismo. Sugere-se, portanto, a necessidade da realização de ações voltadas para os adolescentes visando a prevenção do consumo de álcool, dos transtornos decorrentes, da diminuição de sua prevalência de utilização, bem como, retardo da idade de início deste consumo.

\section{Conclusão}

Verificou-se que os adolescentes de Passos (MG) apresentaram contato precoce com bebidas alcoólicas e alta prevalência de comportamento de beber de risco sendo este maior entre os estudantes do sexo masculino, que não possuíam bom relacionamento com a mãe e que eram trabalhadores.

\section{Colaboradores}

JADB Campos, JC Almeida, PPNS Garcia e JB Faria participaram igualmente de todas as etapas da elaboração do artigo.

\section{Agradecimentos}

À Fundação de Amparo à Pesquisa do Estado de São Paulo (FAPESP) pelo auxílio financeiro concedido. Às escolas e aos estudantes da cidade de Passos (MG) que concordaram em participar do estudo. 


\section{Referências}

1. Room R, Babor T, Rehm J. Alcohol and public health. Lancet 2005; 365(9458):519-530.

2. Centro Brasileiro de Informações sobre Drogas (CEBRID). Universidade de São Paulo. [acessado 2008 out 10]. Disponível em: http://www.unifesp.br/dpsicobio/cebrid/

3. Silveira DX, Moreira FG. Panorama atual de drogas e dependências. São Paulo: Atheneu; 2006.

4. Vizzolto SM. A droga, a escola e a prevenção. Petrópolis: Vozes; 1987.

5. Andersen A, Due P, Holstein BE, Iversen L. Tracking drinking behaviour from age 15-19 years. Addiction 2003; 98(11):1505-1511.

6. Departamento de Adolescência da Sociedade Brasileira de Pediatria. Uso e abuso de álcool na adolescência. Adolescência \& Saúde 2007;4(3):6-59.

7. López JR, Antolín N, Barceló MV, Pérez M, Ballesteros AM, García AL. Consumo de alcohol en los escolares de un área de salud. Hábitos y creencias. Aten Primaria 2001; 27(3):159-165.

8. Gallego MPO, Jiménez MS, Castro FL, Armero MAT. Consumo de alcohol en escolares toledanos: motivos y alternativas. Aten Primaria 2005; 36(6):297-305.

9. Caetano R, Babor TF. Diagnosis of alcohol dependence in epidemiological surveys: an epidemic of youthful alcohol dependence or a case of measurement error? Addiction 2006; 101(Supl. 1):111-114.

10. Galduróz JCF, Noto AR, Fonseca AM, Carlini EA. $V$ Levantamento Nacional sobre o consumo de drogas psicotrópicas entre estudantes do ensino fundamental e médio da rede pública de ensino nas 27 capitais brasileiras - 2004. São Paulo: Centro Brasileiro de Informações sobre Drogas Psicotrópicas/Departamento de Psicobiologia, Universidade Federal de São Paulo; 2005.

11. Vieira DL, Ribeiro M, Romano M, Laranjeira R. Álcool e adolescentes: estudo para implementar políticas municipais. Rev Saude Publica 2007; 41(3): 396-403.

12. Babor TF, Higgins-Bidle JC, Saunders JB, Monteiro MG. AUDIT - The alcohol use disorders identification test. $2^{\text {nd }}$ ed. Geneva: World Health Organization (WHO); 2001.

13. Méndez EB. Uma versão brasileira do AUDIT [dissertação]. Pelotas: Universidade Federal de Pelotas; 1999.

14. Lima CT, Freire CC, Silva APB, Teixeira RM, Farrel $\mathrm{M}$, Prince M. Concurrent and construct validity of the AUDIT in an urban Brazilian sample. Alcohol Alcoholism 2005; 40(6):584-589.

15. Almeida JC, Campos JADB. Desordens devido ao álcool em adolescentes: Confiabilidade de um instrumento de medida. Alim Nutr 2009; 20(3):435-440.

16. Associação Brasileira dos Institutos de Pesquisa de Mercado (ABIPEME). Proposição para um novo critério de classificação socioeconômica. São Paulo: ABIPEME; 1978. [mimeo]

17. Molina SO, Torres DP, Molina SO, Espejo RS. Consumo de alcohol en Estudiantes de secundaria de Córdoba. Enfermería Clínica 2003; 13(4):202-207.
18. Soldera M, Dalgalarrondo P, Filho HRC, Silva CAM. Uso pesado de álcool por estudantes dos ensinos fundamental e médio de escolas centrais e periféricas de Campinas (SP): prevalência e fatores associados. Rev Bras Psiquiatr 2004; 26(3):174-179.

19. Mateos JLC, Luzardo MCM, Riquelme FG. Consumo de alcohol en adolescentes de tres municipios de Lanzarote. Semergen 2004; 30:210-217.

20. Souza DPO, Areco KN, Filho DXS. Álcool e alcoolismo entre adolescentes da rede estadual de ensino de Cuiabá, Mato Grosso. Rev Saude Publica 2005; 39(4):585-592.

21. Vieira DL, Ribeiro M, Laranjeira R. Evidence of association between early alcohol useand risk of later problems. Rev Bras Psiquiatr 2007; 29(3):222-227.

22. McCarty CA, Ebel BE, Garrison MM, Digiuseppe DL, Christakis DA, Rivara FP. Continuity of Binge and Harmful Drinking From Late Adolescence to Early Adulthood. Pediatrics 2004;114(3):714-719. [acessado 2009 ago 14]. Disponível em: http:// www.pediatrics.org/cgi/content/full/114/3/714

23. Vorst HVD, Engels CME, Meeus W, Dekovic M, Leeuwe JV. The role of alcohol-specific socialization in adolescents drinking behaviour. Addict 2005; 100(10):1464-1476.

24. Lama JG, Fernández JRC, León PP. Estudio epidemiológico de comportamientos de riesgo en adolescentes escolarizados de dos poblaciones, semirrural y urbana. Aten Primaria 2002; 30(4):214-219.

25. Oliveira EB, Bittencourt LP, Carmo AC. A importância da família na prevenção do uso de drogas entre crianças e adolescentes: papel materno. SMAD. Rev Eletronica Saude Mental Álcool Drog 2008; 4(2). [acessado 2009 ago 14]. Disponível em: http://pepsic.bvs-psi.org.br/scielo

26. Contrin BC, Carvalho CG, Gouveia N. Comportamento de saúde entre jovens estudantes das redes pública e privada da área metropolitana do Estado de São Paulo. Rev Saude Publica 2000; 34(6):636-645.

27. Pechansky F, Szobot CM, Scivoletto S. Uso de álcool entre adolescentes: conceitos, características epidemiológicas e fatores etiopatogênicos. Rev Bras Psiquiatr 2004; 26(Supl. 1):14-17.

28. Colby SM, Lee CS, Esquerre JL, Smythers CE, Monti PM. Adolescent alcohol misuse: methodological issues for enhancing treatment research. Addiction 2004; 99(Supl. 2):47-69.

29. Laranjeira R, Pinsky I. O alcoolismo. $7^{a}$ ed. São Paulo: Contexto; 2001.

30. Schinkerl M, Minayo MCS. A importância da família no tratamento do abuso de drogas: uma revisão da literatura. Cad Saude Publica 2004; 20(3):649659.

31. Ruiz MR, Andrade D. La família y los factores de riesgo relacionados com el consumo de alcohol y tabaco em los niños y adolescentes (Guayaquil Ecuador). Rev. Latino-am. Enfermagem 2005; 13(Spec. N.):813-818.

32. Silva LVER, Malbergier A, Stempliuk VA, Andrade AG. Fatores associados ao consumo de álcool e drogas entre universitários. Rev Saude Publica 2006; 40(2):280-288. 
33. Dalgalarrondo P, Soldera MA, Correa Filho HR, Silva CAM. Religião e uso de drogas por adolescentes. Rev Bras Psiquiatr 2004; 26(2):82-90.

34. Kozaryn-Okulicz K, Borucka A. Warsaw adolescent alcohol use in a period of social change in Poland: Cluster analyses of five consecutive surveys, 1988 to 2004. Addict Behav 2008; 33(3):439-450.

Artigo apresentado em 22/09/2009

Aprovado em 27/01/2010

Versão final apresentada em 10/02/2010 